\title{
Intraoperative contrast enhanced ultrasound adds some important details to the endovascular aortic aneurysm repair completion control
}

\author{
Chiara Mascoli, Gianluca Faggioli, Enrico Gallitto, Rodolfo Pini, Mauro Gargiulo \\ Policlinico S. Orsola Malpighi, Vascular Surgery, University of Bologna, DIMES, Bologna, Italy \\ Correspondence to: Chiara Mascoli, MD. Vascular Surgery, University of Bologna, Azienda Policlinico S. Orsola-Malpighi, Padiglione 23, I piano, Via \\ Massarenti 9, 40138 Bologna, Italy. Email: chiara.ma@yahoo.it. \\ Provenance: This is an invited article commissioned by the Academic Editor Dr. Zhongzhi Jia (Department of Interventional and Vascular Surgery, \\ The Affiliated Changzhou No. 2 People's Hospital of Nanjing Medical University, Changzhou, China). \\ Comment on: Bianchini Massoni C, Perini P, Fanelli M, et al. Intraoperative contrast-enhanced ultrasound for early diagnosis of endoleaks during \\ endovascular abdominal aortic aneurysm repair. J Vasc Surg 2019. [Epub ahead of print].
}

Submitted Nov 13, 2019. Accepted for publication Nov 18, 2019.

doi: 10.21037/atm.2019.11.120

View this article at: http://dx.doi.org/10.21037/atm.2019.11.120

Contrast enhanced ultrasound (CEUS) has been widely accepted as an effective and reproducible diagnostic tool in the follow-up after endovascular aortic aneurysm repair (EVAR) for a long time. It is particularly useful in the detection of endoleaks (EL) since is a real-time nonionizing radiation examination, with no nephrotoxic effect with comparable or even superior diagnostic performance compared with standard angiography $(1,2)$.

Several studies have shown a higher sensitivity for low flow endoleaks of this diagnostic method if compared with the most commonly used duplex ultrasound and computed tomography angiography (1-4). Thanks to these experiences, CEUS is currently considered a necessary tool for the EVAR follow-up. Nevertheless, very few papers are available in the literature on its intraoperative use as a diagnostic and interventional method $(5,6)$.

This gives added value to the study by Bianchini Massoni et al. (7), that reported the intraoperative use of CEUS compared with digital subtraction angiography performed in two orthogonal projection (2DSA), for the detection of endoleaks.

Moreover, the authors investigated the utility of CEUS for intraoperative adjunctive procedures.

The intraoperative CEUS examination was easily performed under sterile condition in 60 cases, before the end of the EVAR procedure. Considering any type of EL detection, the two diagnostic methods, i.e., CEUS and
DSA showed a "fair" agreement (Cohen's kappa $=0.250$ ), with 2DSA revealing 11 ELs and CEUS 25 ELs. CEUS showed 17 Els (28\%) undetected at 2DSA: 15 type II ELs and 2 type IA ELs (7). Therefore, the intraoperative CEUS showed to be useful in the detection of type II and low flow type I A endoleaks, not easily visible by DSA (7).

More interestingly, this experience highlighted the use of CEUS as a guide to optimize the preventive embolization of type II endoleaks. As a matter of fact, among the 23 cases of type II EL detected by CEUS, 4 (17\%) were classified preoperatively as at high risk for persistent type II EL; thus, sac embolization was effectively performed intraoperatively under CEUS control with type II Els resolution in all the cases (7).

The authors performed the intraoperative sac embolization only in selected cases, preoperatively identified as at risk for type II persistent EL. Only in those cases, a 5F catheter was placed in the aneurysm sac in order to perform the embolization with coils. Data in the literature show the effectiveness of this technique in reducing the incidence of persistent type II endoleaks in selective cases, even thou the most effective embolization method is yet to be defined (8).

The authors suggest that intraoperative CEUS is effective in evaluating the efficacy of this preventive technique, establishing the number of necessary coils, without further radio exposure or use of contrast medium.

The study by Bianchini Massoni et al. (7), despite the 
undisputed limits due to the low number of patients and the lack of follow-up data, gives new insights in the use of CEUS.

\section{Acknowledgments}

None.

\section{Footnote}

Conflicts of Interest: The authors have no conflicts of interest to declare.

Ethical Statement: The authors are accountable for all aspects of the work in ensuring that questions related to the accuracy or integrity of any part of the work are appropriately investigated and resolved.

\section{References}

1. Mauro R, Maioli F, Freyrie A, et al. Is CEUS a valid alternative to CTA in endoleak's detection? J Vasc Endovasc Surg 2010;17:253-8.

2. Perini P, Sediri I, Midulla M, et al. Single-centre prospective comparison between contrast-enhanced ultrasound and computed tomography angiography after EVAR. Eur J Vasc Endovasc Surg 2011;42:797-802.

Cite this article as: Mascoli C, Faggioli G, Gallitto E, Pini R, Gargiulo M. Intraoperative contrast enhanced ultrasound adds some important details to the endovascular aortic aneurysm repair completion control. Ann Transl Med 2019;7(Suppl 8):S280. doi: 10.21037/atm.2019.11.120
3. Gargiulo M, Gallitto E, Serra C, et al. Could fourdimensional contrast-enhanced ultrasound replace computed tomography angiography during follow up of fenestrated endografts? Results of a preliminary experience. Eur J Vasc Endovasc Surg 2014;48:536-42.

4. Mascoli C, Faggioli G, Gallitto E, et al. The Assessment of Carbon Dioxide Automated Angiography in Type II Endoleaks Detection: Comparison with ContrastEnhanced Ultrasound. Contrast Media Mol Imaging 2018;2018:7647165.

5. Hertault A, Maurel B, Pontana F, et al. Benefits of completion $3 \mathrm{D}$ angiography associated with contrast enhanced ultrasound to assess technical success after EVAR. Eur J Vasc Endovasc Surg 2015;49:541-8.

6. Ormesher DC, Lowe C, Sedgwick N, et al. Use of three-dimensional contrast-enhanced duplex ultrasound imaging during endovascular aneurysm repair. J Vasc Surg 2014;60:1468-72.

7. Bianchini Massoni C, Perini P, Fanelli M, et al. Intraoperative contrast-enhanced ultrasound for early diagnosis of endoleaks during endovascular abdominal aortic aneurysm repair. J Vasc Surg 2019. [Epub ahead of print].

8. Mascoli C, Freyrie A, Gargiulo M, et al. Selective Intraprocedural AAA sac Embolization During EVAR Reduces the Rate of Type II Endoleak. Eur J Vasc Endovasc Surg 2016;51:632-9. 